Research Paper

\title{
Maximizing mechanical properties and minimizing support material of PolyJet fabricated 3D lattice structures
}

\author{
Wenfeng Liu ${ }^{\mathrm{a}, \mathrm{b}}$, Hongwei Song ${ }^{\mathrm{a}, \mathrm{b}, *}$, Chenguang Huang ${ }^{\mathrm{a}, \mathrm{b}}$ \\ ${ }^{a}$ Key Laboratory for Mechanics in Fluid Solid Coupling Systems, Institute of Mechanics, Chinese Academy of Sciences, Beijing, 100190, China \\ ${ }^{\mathrm{b}}$ School of Engineering Science, University of Chinese Academy of Sciences, Beijing, 100049, China
}

\section{A R T I C L E I N F O}

\section{Keywords:}

Anisotropy

PolyJet technology

lattice structures

Snap-fit

Strength

\begin{abstract}
A B S T R A C T
Anisotropy of mechanical properties and support material removal are the two main problems when fabricating 3D lattice structures by integrated printing via additive manufacturing (AM) technology. Aiming at these two problems, a snap-fit method is introduced into PolyJet technology to fabricate polymer lattice structures with four typical configurations, namely BCC, BCC-Z, FCC and octet. Printing materials and printing time in this novel method are both reduced by over $80 \%$ compared to the conventional printing method. Uniaxial compression tests indicate that both the strengths and energy absorptions of the four kinds of snap-fitted lattices are increased by over $100 \%$ compared to the integrated counterparts. The effect of strut thickness on compressive responses of the snap-fitted and integrated lattices is investigated. With the decrease of strut thickness, the advantage in the strength of the snap-fitted lattices becomes more obvious compared to the integrated counterparts. Ideal maximum strength models based on yielding, elastic buckling and inelastic buckling are developed and are able to predict the compressive peak strengths of the snap-fitted PolyJet lattices. This study opens up an avenue for the fabrication of large scale 3D printed lattice structures with optimal mechanical properties and without support material removal problem.
\end{abstract}

\section{Introduction}

Additively manufactured 3D lattice structures have attracted great interest in recent years due to their extensive application prospect in ultralight load-bearing structures [1-3], impact energy absorbers [4,5], biomedical implants $[6,7]$ and so on. The most commonly reported AM technology to fabricate metallic lattice structures is the selective laser melting (SLM). With this method, high strength can be achieved and support material can be easily removed. SLM has been used to fabricate octet, BCC, cubic and many other lattices, and the static compressive and impact behaviors were studied [8-11]. Fused deposition modeling (FDM) is one of the most widely used AM technologies to fabricate thermal plastic lattices, owing to its simple printing process and low cost. Disordered lattice materials [12] and BCC-Z lattice [13] with Polylactic acid (PLA), octet and octahedral lattices with a carbon fiber reinforced composite of PLA [14] and cubic-center lattice with Acrylonitrile Butadiene Styrene (ABS) [15] were manufactured by FDM. PolyJet is a powerful AM technology that has high printing accuracy and multi-material capacity. Egan et al. [16,17] investigated polymer lattices printed by PolyJet technique for tissue engineering. Mueller et al. [18] added stepwise graded core-shell struts to bending dominated lattices fabricated by PolyJet to increase energy absorption.

All these lattice structures mentioned in the above references are printed integrally. However, there are two common problems for integrally printed 3D lattice structures. One is anisotropy resulted from build direction of printed parts, which is one of the most influential factors on mechanical properties and almost exists in all AM technologies [15,19-21]. The difference of build directions of struts in the integrated lattice structures makes their mechanical properties far from optimal. A common strategy to improve the mechanical performance of lattice structures is changing the build direction of the whole lattice part according to a given loading direction [21-24]. However, strengthening in one direction generally results in weakening in other directions of the 3D lattice structures. In addition, changing build direction tends to increase the difficulty of printing and printing time and consume more support materials. Another problem is the removal of support material. Removing support material from complex cellular structures is always a challenge, especially for the FDM and PolyJet techniques. A large amount of support material is needed to assist modeling during the printing process due to the high porosity of the

\footnotetext{
* Corresponding author at: Key Laboratory for Mechanics in Fluid Solid Coupling Systems, Institute of Mechanics, Chinese Academy of Sciences, Beijing, 100190, China.

E-mail address: songhw@imech.ac.cn (H. Song).
} 
lattice. The presence of support material not only significantly increases the printing cost but also increases the difficulty of removing it. In many cases, the support material is difficult or even impossible to be removed completely, especially for the lattice with complex configurations and a large number of elements. Aiming at these two problems, Liu et.al [25]. introduced a snap-fit assembly method into the FDM technique to fabricate the BCC lattice structures with all extruded filaments deposited along the length direction of the strut. The lattice structures fabricated in this way not only achieve improved mechanical performance but need no support material during the printing process.

The anisotropy and support material removing problems are more obvious in PolyJet printed lattices. Here, we explore the application of a snap-fit assembly method for fabricating the lattice structures with four kinds of typical configurations from PolyJet printed trusses. Mechanical responses of the four kinds of snap-fitted lattices under compression have been experimentally investigated and compared with the integrally printed lattices. This study opens up an avenue for the fabrication of large scale 3D printed lattice structures with optimal mechanical properties and without support material removal problem.

\section{Design and fabrication}

PolyJet is a powerful AM technology that layers of curable liquid photopolymer are jetted onto a build tray and cured by ultra-violet (UV) light, creating smooth, precise and accurate models [26]. The conventional integrated fabricating process of lattice structures using this technology are shown in Fig. 1(a). The entire lattice sample with support material is printed firstly as shown in Fig.1 (b), and then a blade is used to remove the external support material and a water-jet is used to remove the internal support material, leaving the final lattice part as shown in Fig.1 (c).

Anisotropy of mechanical properties resulted from build orientation also exists in PolyJet technology. Previous studies revealed that the highest strength and failure strain of a PloyJet model is reached at the in-plane orientations (X-Y plane). Transversally isotropic properties are obtained in the $\mathrm{X}-\mathrm{Y}$ plane, but any involvement of $\mathrm{Z}$ quickly reduces the strength and fracture strain $[19,27,28]$. It is worth mentioning that although the strength and modulus in the $\mathrm{X}-\mathrm{Y}$ plane are assumed isotropic, actually, the strength and elastic modulus of the sample printed in the $\mathrm{X}$ direction are slightly larger than the other direction in the $\mathrm{X}-\mathrm{Y}$ plane $[19,28]$. The second-largest strength and elastic modulus are reached in the $\mathrm{Y}$ direction.

In order to make all the struts in 3D lattices have the best mechanical properties, a snap-fit assembly method is introduced to design and fabricate the lattices with four kinds of typical configurations, namely BCC, BCC-Z, FCC and octet. The fabrication process and the geometric parameters of the snap-fitted BCC, BCC-Z, FCC and octet lattices are shown in Fig. 2 (a), (b), (c) and (d), respectively. First, the 2D lattice truss patterns and intermediate layer patterns were printed on the build tray along the direction shown in the upper-right items of each figure in Fig. 2. Note that all the struts in the lattices have a square cross section and the node width, $b$, is three times the strut thickness, $t$. The intermediate layer face sheets are printed with through holes, and the up and bottom face sheets are printed with slots of $t / 2$ depth such that the nodes of the up and bottom truss core could be counter-sunk into the face sheets. Second, the printed truss patterns of each configuration are then orthogonally snap-fitted into each other to produce a single layer truss core. Finally, the single truss layer planes are then snap-fitted into the slots of the bottom and intermediate face sheet and bonded at the nodal regions of the assembled structures using an epoxy adhesive (AB Glue, 302, Gleihow ${ }^{\circledR}$ Brand). The process is repeated to form the multilayer lattice structures as shown on the left side of each figure in Fig. 2. Note that a small gap is reserved in the center slot of half of the FCC trusses to allow for assembly. The slot tolerance in the nodal regions of all trusses is controlled at $80 \mu \mathrm{m}$ to enable smooth and stable matching between each assembled part. A glue gun is used to quickly and evenly apply glue at the nodes.

The build directions of each strut of the four kinds of snap-fitted and integrated lattices are shown in Fig. 3. Three possible build directions of the struts in lattices, $0^{\circ}, 45^{\circ}$ and $90^{\circ}$, are represented by three kinds of colors, green, yellow and red, respectively. Fig. 3 shows that all the struts of four kinds of lattices fabricated by the snap-fit method are printed in the $\mathrm{X}-\mathrm{Y}$ plane and have the best mechanical properties. While two build directions, $0^{\circ}$ and $45^{\circ}$, exist in the integrated BCC, FCC and octet lattices and three build directions exist in the integrated BCC$\mathrm{Z}$ lattice.

Photographs of the $2 \times 2 \times 2$ cells lattice structures with strut thickness, $t=2 \mathrm{~mm}$, and strut slenderness ratio, $t / l=0.011$, of these four kinds of configurations and their unit cells fabricated by the proposed method are shown in Fig. 4. The heights of the unit cell and $2 \times 2 \times 2$ cells lattices are maintained at $34 \mathrm{~mm}$ and $66 \mathrm{~mm}$, respectively. The integrally printed lattices with the same geometric parameters are fabricated as counterparts. All the samples are printed on a Stratasys Objet30pro printer with a layer thickness of $0.016 \mathrm{~mm}$ and a resolution of $600 \mathrm{dpi}$ in $\mathrm{X}$ and $\mathrm{Y}$ direction, and $900 \mathrm{dpi}$ in the $\mathrm{Z}$ direction. The samples are printed with VeroWhitePlus (RGD835) model and SUP706 support material. The snap-fitted truss surfaces are not covered in support material to enable smooth matching and ensure bonding strength. In contrast, the integrated lattice surfaces are covered in support materials to ensure a continuous smooth surface.

The cost of the PolyJet printed lattices mainly comes from the consumption of printing materials and printing time. Comparisons of

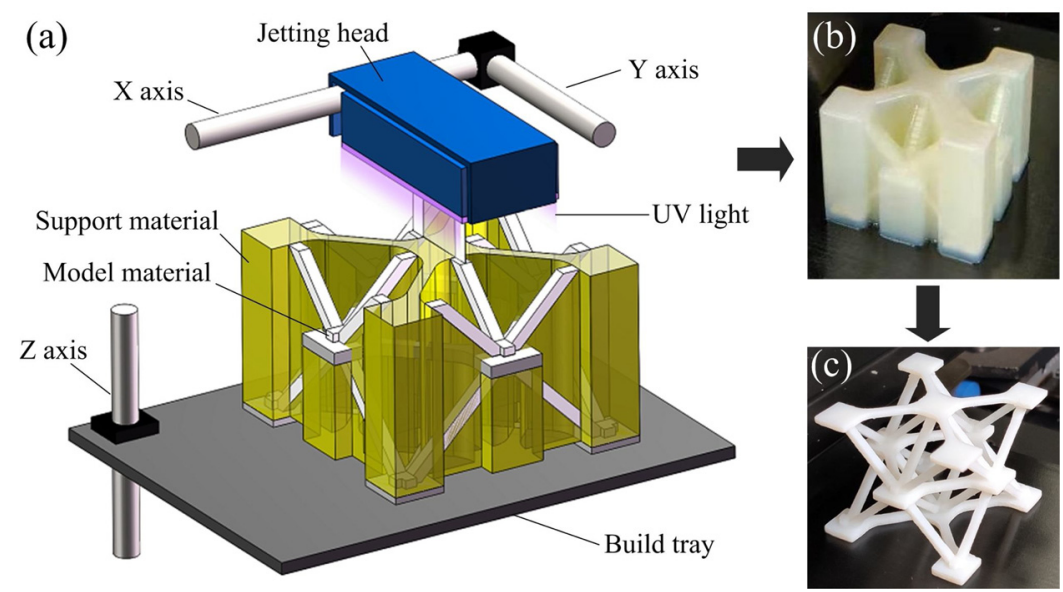

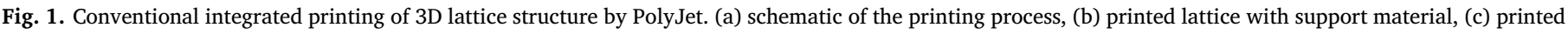
lattice after removing support material. 

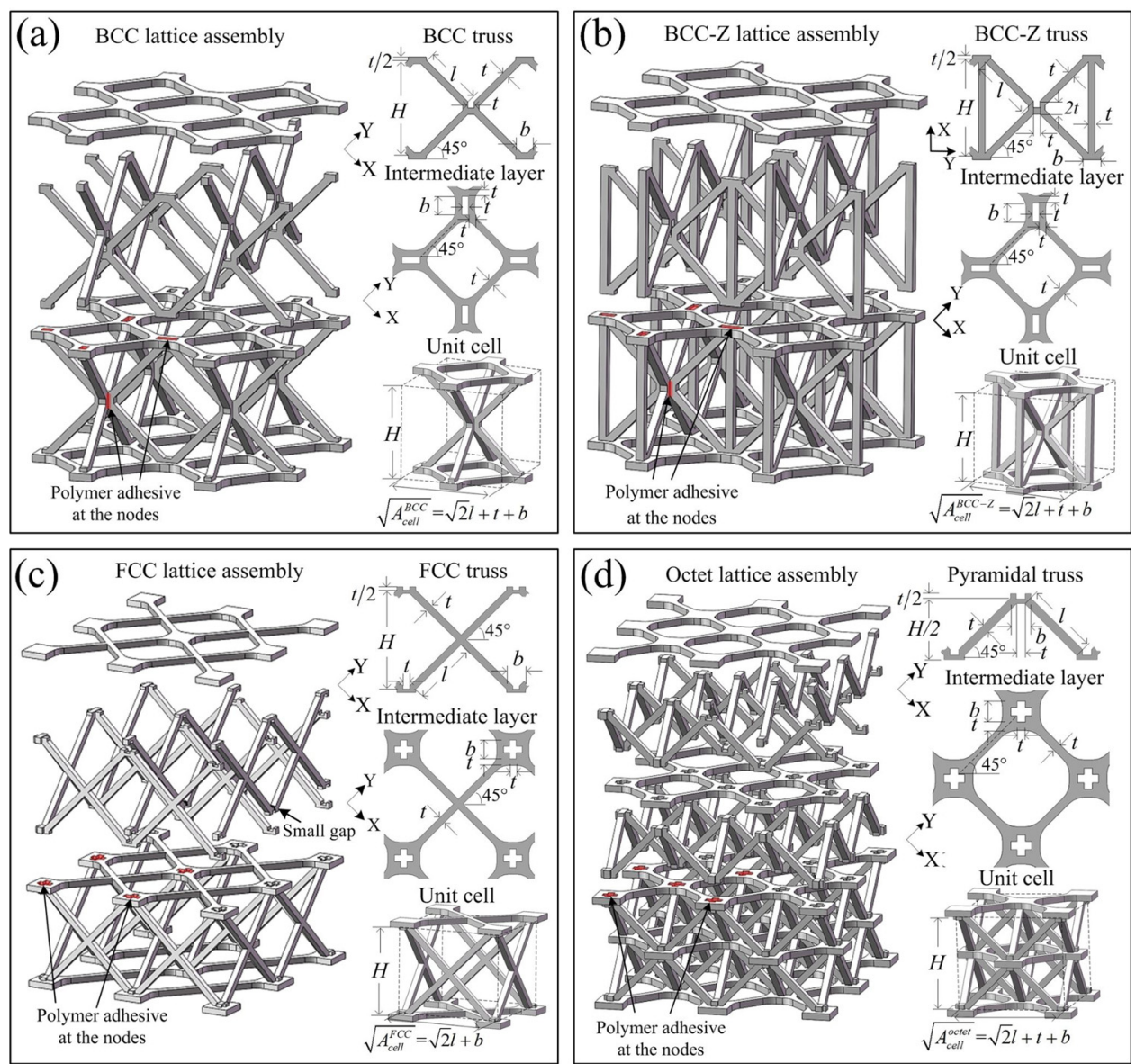

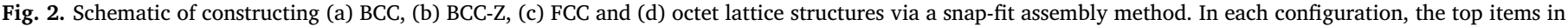
the right column are the disassembled $2 \mathrm{D}$ truss patterns and their printing direction in the X-Y plane, the bottom item in the right column is the unit cell.

consumption of printing materials (including model and support materials) and printing time between the snap-fitted and integrated $2 \times 2 \times 2$ cells lattices are shown in Fig. 5(a) and (b), respectively. The total consuming materials of the snap-fitted BCC, BCC-Z, FCC and octet lattice structures are reduced by $87.3 \%, 84.9 \%, 86.9 \%$ and $83.4 \%$, respectively, compared to the integrated printing counterparts. These dramatic reductions mainly result from two aspects. One is from support material, which is the main consumption of printing materials in the integrated lattices. In contrast, the support material is not needed to assist modeling in the snap-fit assembly method. Another is from the printing process. The printer will clear the rest materials in printing head into the waste container at a regular interval (about every 50 layers) to ensure the printing quality during the printing process. Since the layer thickness is small $(0.016 \mathrm{~mm})$, this consumption will be considerable when the height of the printed model is large.

The printing time of the snap-fitted BCC, BCC-Z, FCC and octet lattices is reduced by $88.1 \%, 90.2 \%, 88.1 \%$ and $79.0 \%$, respectively, compared to the integrated counterparts. The printing time is highly dependent on the model height. The transition from 3D lattice to 2D trusses considerably reduces the height of the printed model and minimizes the number of printing head movements and cleaning, therefore dramatically reducing the printing time of the snap-fitted lattices. On the other hand, more area of build tray is also needed in the snap-fit method since the 3D lattice is disassembled into 2D truss patterns. The required area of the build tray and printing time in the snapfit method increase with the complexity and volume of the lattices. The

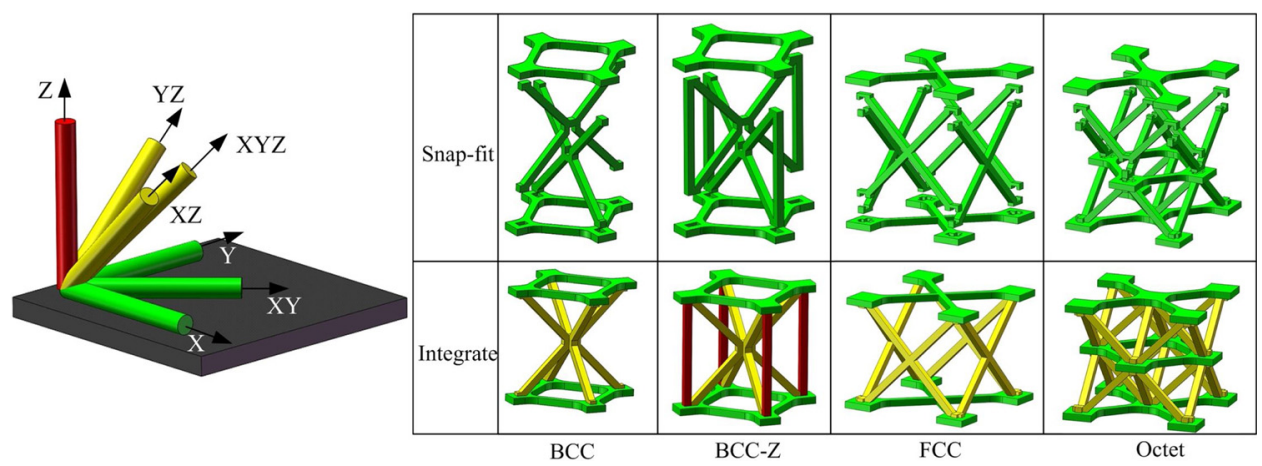

Fig. 3. Build directions of printing struts, Green: $0^{\circ}$, Yellow: $45^{\circ}$, Red: $90^{\circ}$, and the build directions in four kinds of lattice configurations fabricated by snap-fit method (top) and conventional integrate method (bottom), respectively. 


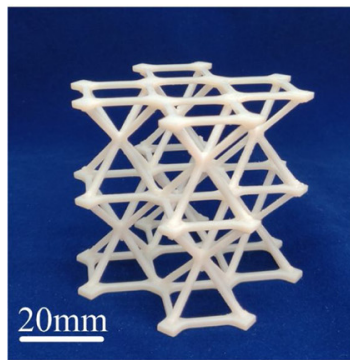

BCC lattice

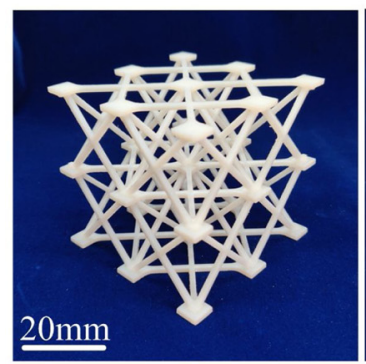

FCC lattice

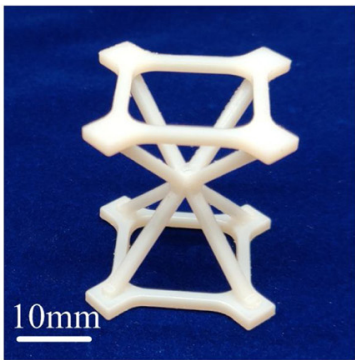

Unit cell

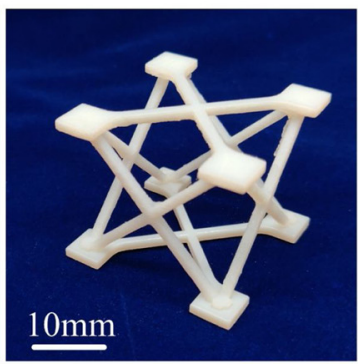

Unit cell

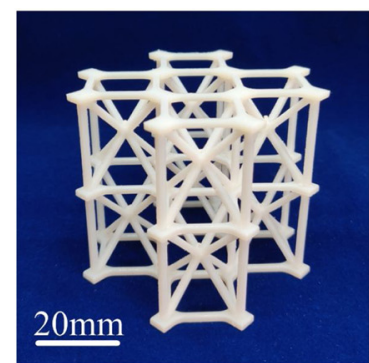

BCC-Z lattice

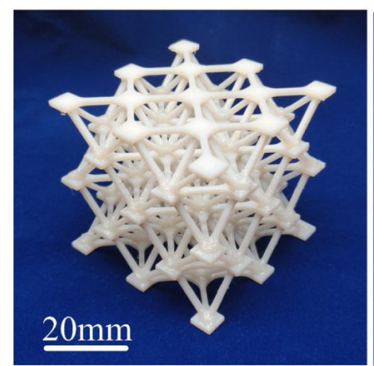

Octet lattice

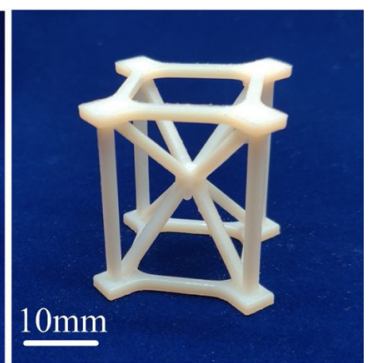

Unit cell

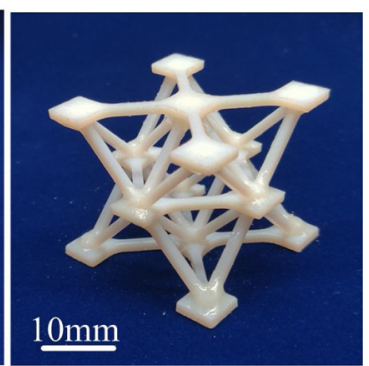

Unit cell

Fig. 4. Photographs of the fabricated BCC, BCC-Z, FCC, and octet lattices and their unit cells via snap-fit assembly method.

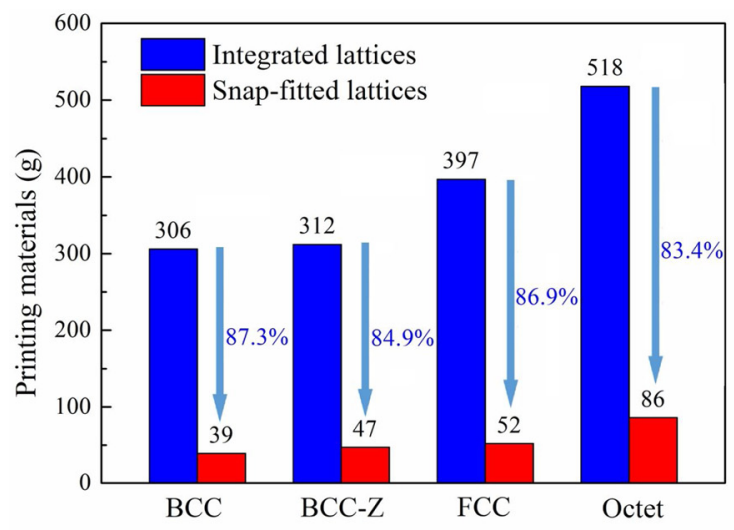

(a)

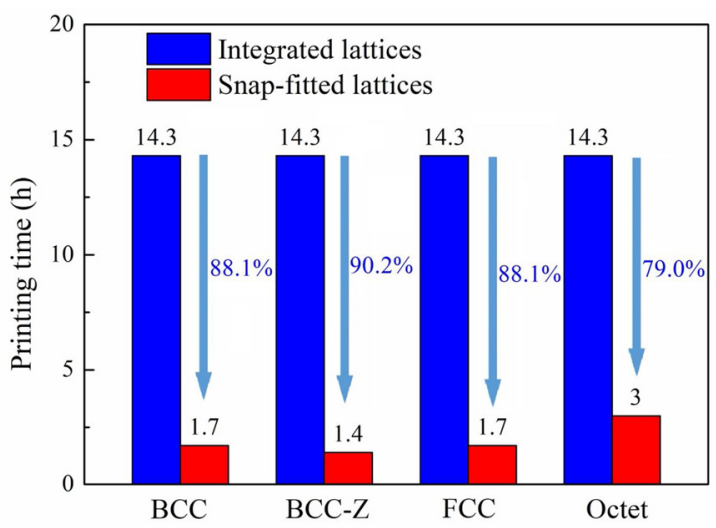

(b)

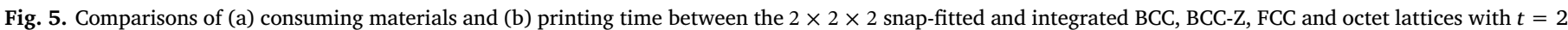
$\mathrm{mm}$.

octet lattice has the most complexity in the four kinds of lattices. Twice the area of the build tray $(294 \mathrm{~mm} \times 192 \mathrm{~mm})$ is needed to print the truss patterns of the octet lattice, taking almost twice as long as the other three kinds of snap-fitted lattices as shown in Fig. 5(b).

The assembling and gluing time of the snap-fitted $2 \times 2 \times 2$ cells BCC, BCC-Z, FCC and octet lattices is around $0.5 \mathrm{~h}, 0.5 \mathrm{~h}, 0.5 \mathrm{~h}$ and $1 \mathrm{~h}$, respectively. All the assembled lattices will rest for over $12 \mathrm{~h}$ to assure the strength of the adhesive. In contrast, the support material removing time of the integrated $2 \times 2 \times 2$ cells BCC, BCC-Z, FCC and octet lattices is around $1.5 \mathrm{~h}, 1.5 \mathrm{~h}, 1.5 \mathrm{~h}$ and $2 \mathrm{~h}$, respectively. Once the support material is removed by the water-jet, the integrated lattices are left to dry overnight (about $12 \mathrm{~h}$ ).

The comparisons of the printing materials, printing time and postprocessing time between the snap-fitted lattices and integrated lattices indicate that the snap-fit method not only significantly reduces fabricating cost of the lattices, but also makes the post-processing more efficient. The difficulty to remove support material increases with the number of lattice elements and joint connectivity. When these parameters are large enough, it is nearly impossible to remove the support material completely by mechanical methods. The integrated octet lattice has the largest difficulty and consumes the longest time to remove the support material in these four kinds of configurations because of the highest joint connectivity.

\section{Experiments}

To validate the benefit on mechanical properties of the snap-fitted PolyJet lattices, out-of-plane compressive tests are conducted on both snap-fitted and integrated lattices of different configurations (Fig. 6). All the experiments are conducted on a uniaxial testing machine (SUST CMT5205) at an applied nominal displacement rate of $2 \mathrm{~mm} / \mathrm{min}$.

\subsection{Compressive responses of multi-cell lattices}

The deformation behaviors and the macroscopic stress-strain curves of the $2 \times 2 \times 2$ cells BCC, BCC-Z, FCC and octet lattices fabricated by the integrated and snap-fitted method are shown in Fig. 6 (a), (b), (c) and (d), respectively. The integrated BCC lattice and the snap-fitted BCC lattice reach their initial peak strengths of $0.128 \mathrm{MPa}$ and 0.259 MPa at strains of 0.028 and 0.04 , respectively. Then the stress drops rapidly due to the buckling of the struts, as shown in Fig. 6 (a). The initial failure occurs at the bottom layer in the integrated BCC lattice 
Integrate

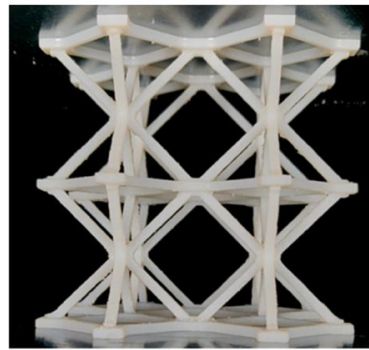

$\varepsilon=0.00$
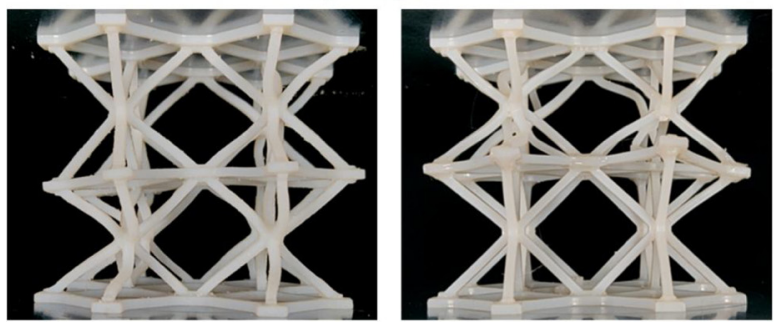

$\varepsilon=0.080$
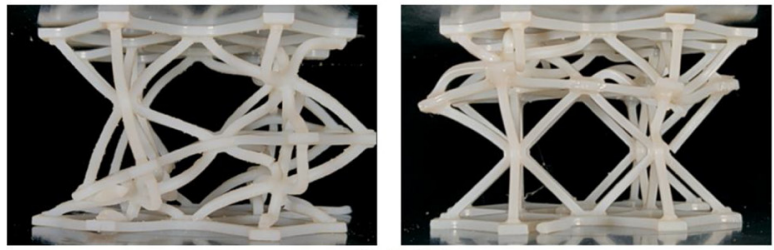

$\varepsilon=0.300$
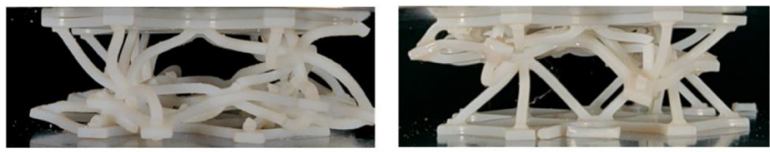

$\varepsilon=0.600$

$30 \mathrm{~mm}$

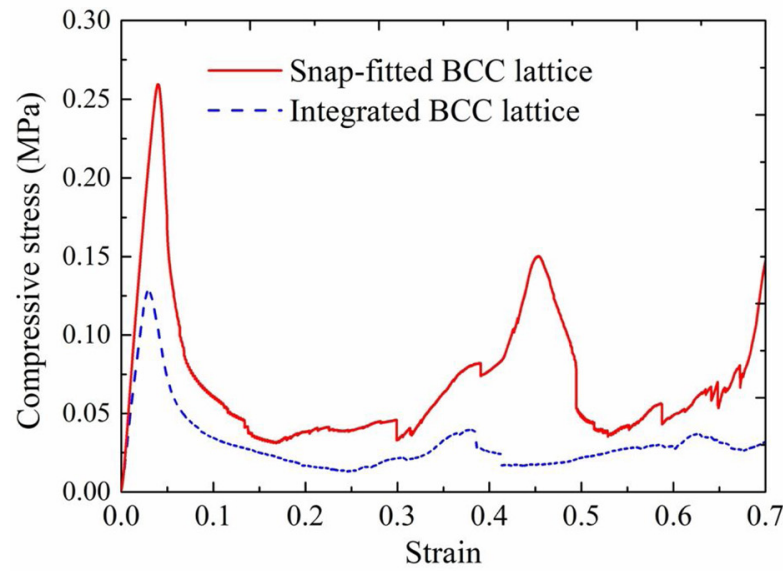

(a)
Integrate

Snap-fit
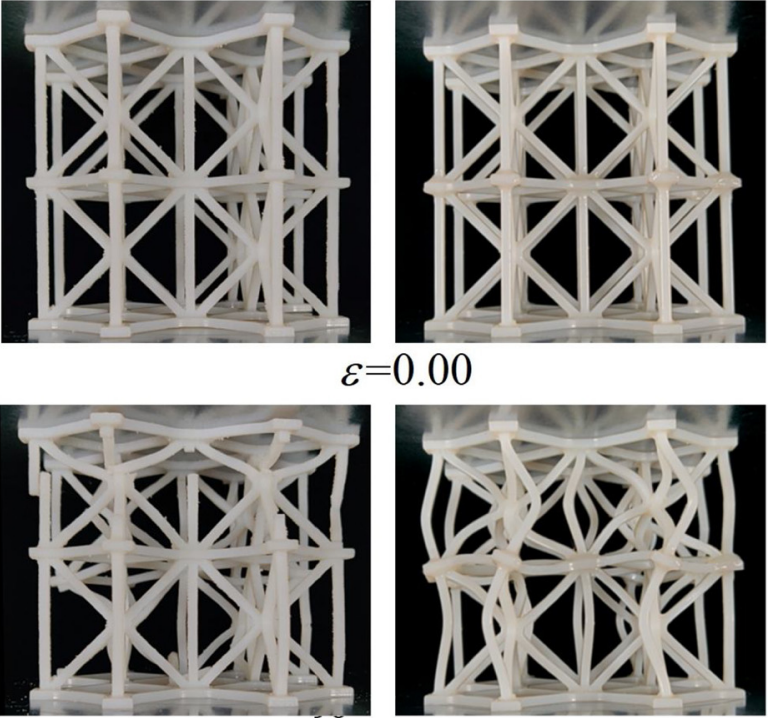

$\varepsilon=0.080$

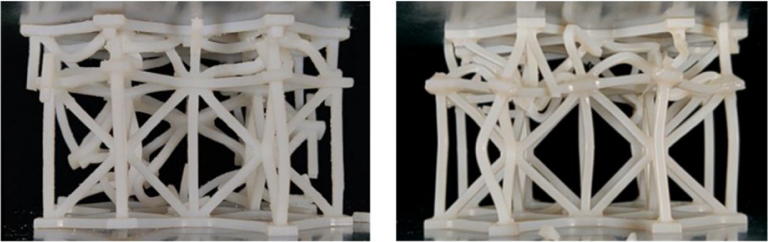

$\varepsilon=0.300$
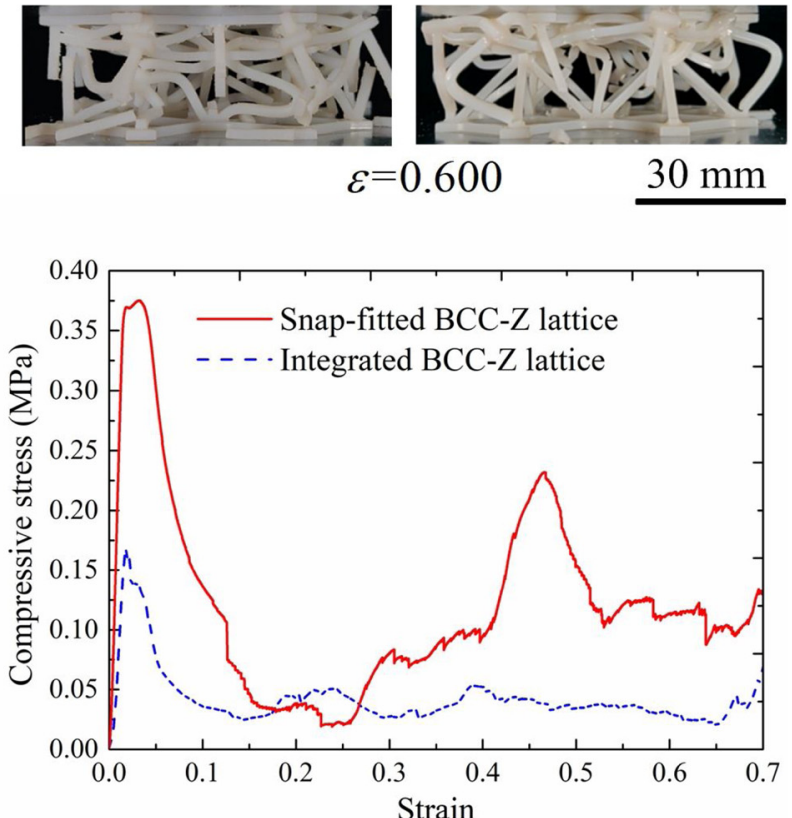

(b)

Fig. 6. Comparisons of deformation behaviors and stress-strain responses of snap-fitted and integrated PolyJet lattices. (a) BCC lattice, (b) BCC-Z lattice, (c) FCC lattice and (d) octet lattice.

and at the upper layer in the snap-fitted lattice. In the process of the bottom layer failure, the upper layer begins to fail for the integrated BCC lattice, owing to the slope of horizontal intermediate struts, resulting in a long and low plateau region until the end strain of 0.7 in the stress-strain curve. By contrast, the bottom layer of the snap-fitted BCC lattice does not fail until the upper layer crushed completely, resulting in the second obvious peak value of $0.15 \mathrm{MPa}$ at a strain of 0.453 in the stress-strain curve. Fig. 6(b) shows the compressive behaviors of the integrated and snap-fitted BCC-Z lattices. Negligible deformation of the struts is observed in the integrated BCC-Z lattice structures until catastrophic strut failure is initiated in the vertical struts. The catastrophic failure results in an immediate stress drop when it reaches the peak 
Integrate

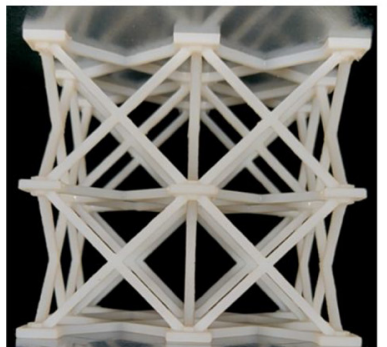

$\varepsilon=0.00$
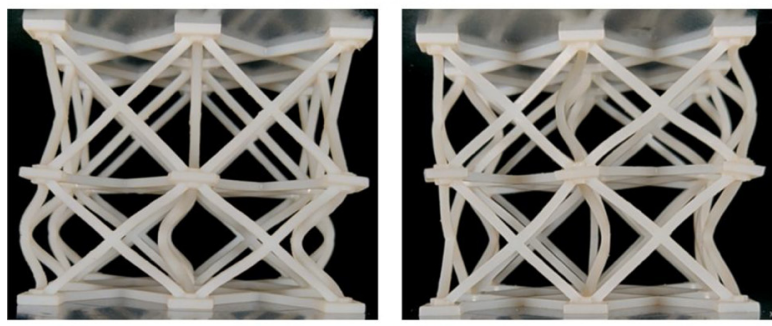

$\varepsilon=0.080$
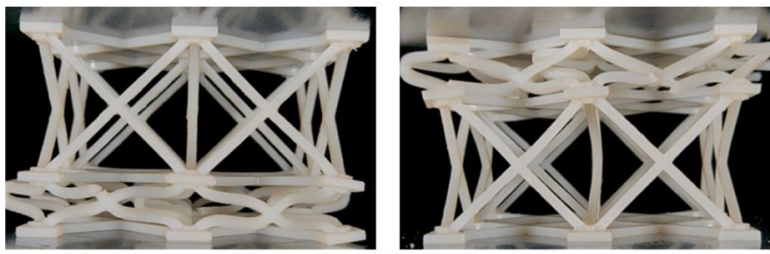

$\varepsilon=0.300$
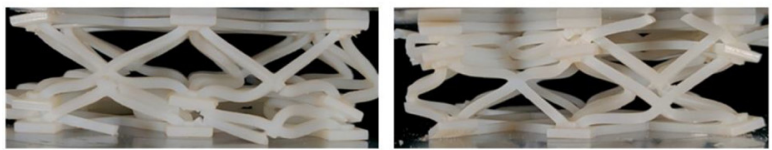

$\varepsilon=0.600$

$30 \mathrm{~mm}$

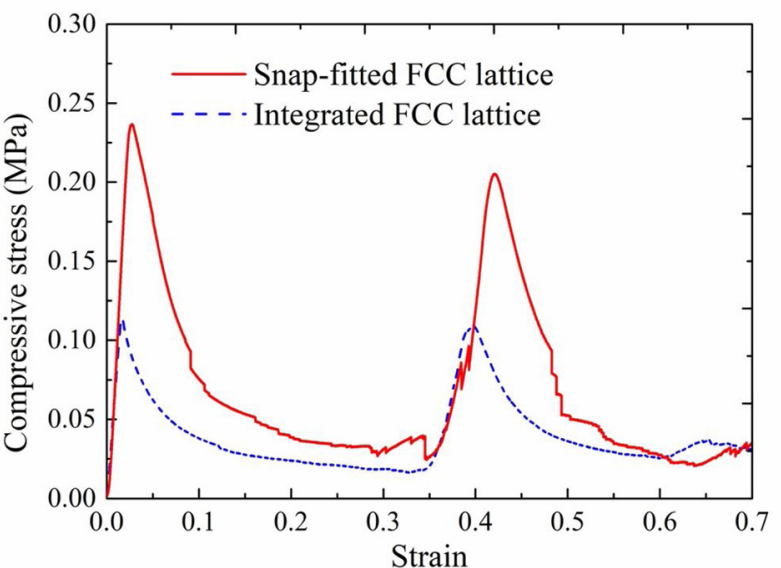

(c)
Integrate
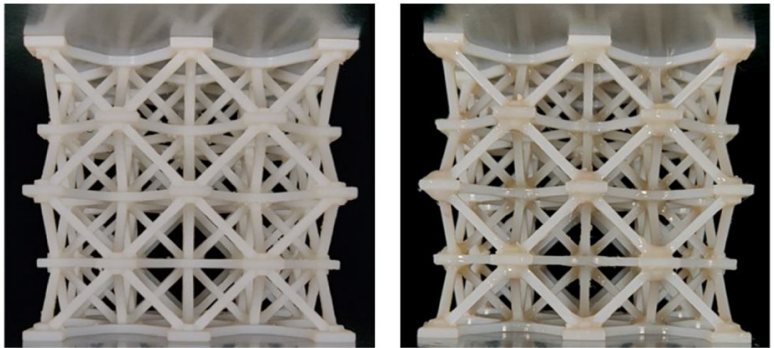

$\varepsilon=0.00$

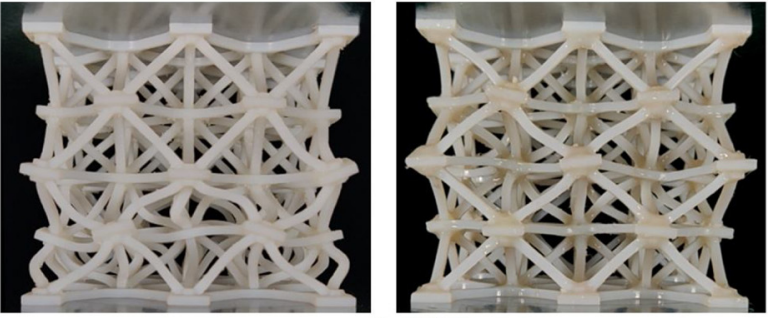

$\varepsilon=0.080$

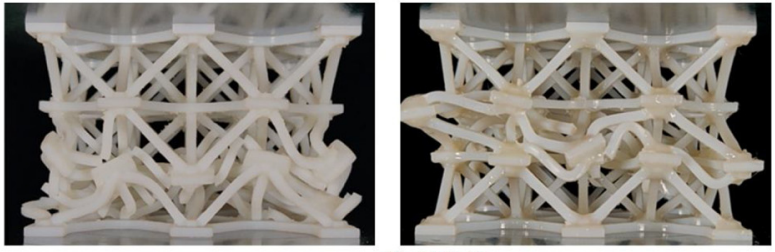

$\varepsilon=0.300$
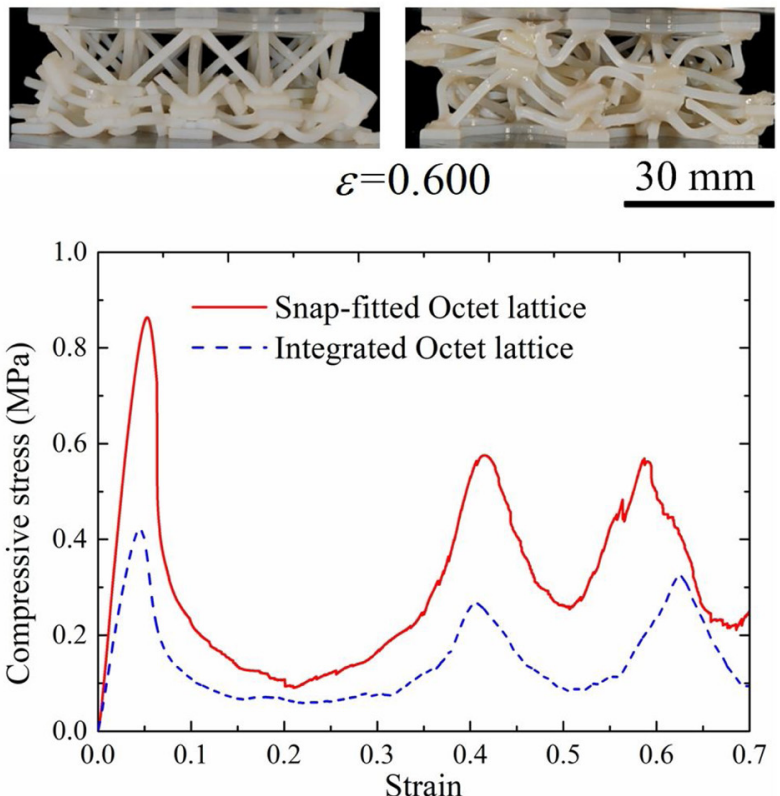

(d)

Fig. 6. (continued)

value of $0.169 \mathrm{MPa}$ at a strain of 0.018 . Several cells in both the upper and bottom layers in the integrated BCC-Z lattice fail simultaneously under compression, resulting in a long and low plateau region in the stress-strain curve. By contrast, the compressive stress of the snap-fitted BCC-Z lattice reaches its initial peak value of $0.372 \mathrm{MPa}$ at a strain of 0.019 , followed by the buckling deformation of the vertical struts and a rapid drop in stress. After a complete compression of the upper layer, the bottom layer begins to fail, leading to the second peak value of
$0.232 \mathrm{MPa}$ at a strain of 0.466 in the stress-strain curve. In Fig. 6(c), the integrated FCC lattice and the snap-fitted FCC lattice reach their initial peak strengths of $0.128 \mathrm{MPa}$ and $0.259 \mathrm{MPa}$ at strains of 0.017 and 0.028 , respectively. Failure of the second layer results in a second peak value of $0.101 \mathrm{MPa}$ at a strain of 0.396 in the integrated FCC lattice, and of $0.205 \mathrm{MPa}$ at a strain of 0.420 in the snap-fitted FCC lattice. In Fig. 6 (d), three peak values are observed in the stress-strain curves of both the integrated and snap-fitted octet lattices. The compressive stress 


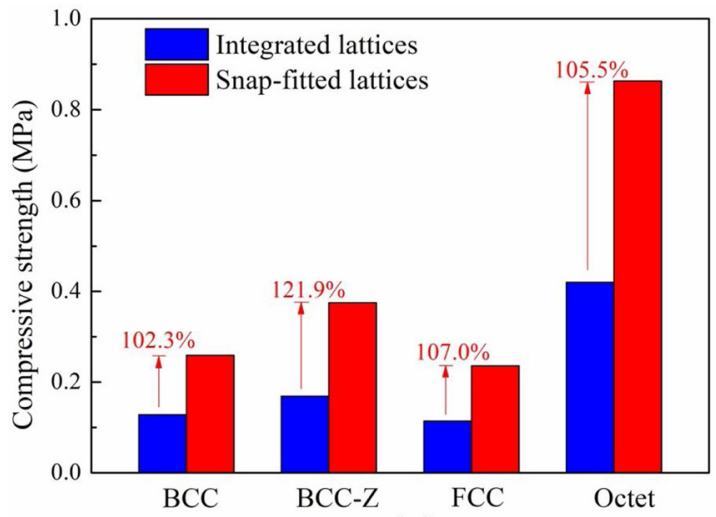

(a)

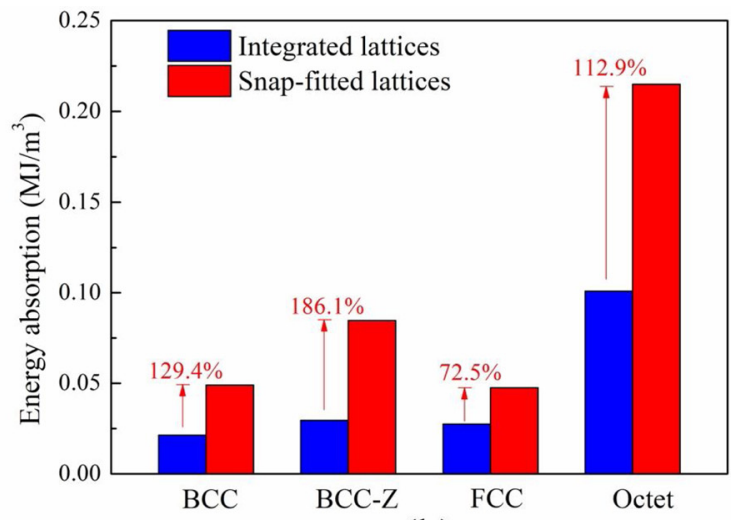

(b)

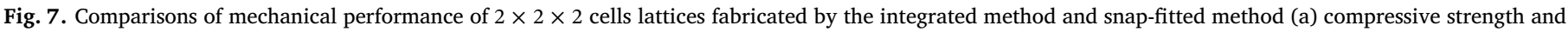
(b) specific energy absorption.

reaches the initial peak value of $0.42 \mathrm{MPa}$ at a strain of 0.045 , the second peak value of $0.267 \mathrm{MPa}$ at a strain of 0.406 , and the third peak value of $0.323 \mathrm{MPa}$ at a strain of 0.627 for integrated octet lattice. The first failure in the integrated octet lattice occurs at the bottom layer and the remaining layers fail from bottom to top layer by layer. By contrast, the compressive stress in the snap-fitted octet lattice reaches the initial peak value of $0.863 \mathrm{MPa}$ at a strain of 0.052 , the second peak value of $0.576 \mathrm{MPa}$ at a strain of 0.416 , and the third peak value of $0.569 \mathrm{MPa}$ at a strain of 0.587 . The lattice fails progressively, i.e., the interlayer, the bottom and top layers fail successively after a complete crush of the foregoing layer.

Comparisons of the initial peak strengths between the snap-fitted lattices and integrated lattices are shown in Fig. 7(a). The compressive strengths of the snap-fitted BCC, BCC-Z, FCC and octet lattices increase by $102.3 \%, 121.9 \%, 107.0 \%$ and $105.5 \%$, respectively, compared to the integrated counterparts.

The specific energy absorption, i.e., energy absorbed per unit volume, $W$, is defined as

$W=\int_{0}^{\varepsilon_{D}} \sigma(\varepsilon) d \varepsilon$

Where $\varepsilon_{D}$ is the densification strain (measured at 0.7 in the four kinds of lattices). Fig. 7(b) shows comparisons of energy absorption between snap-fitted and integrated lattices. The specific energy absorption of the snap-fitted BCC, BCC-Z, FCC and octet lattices increases by $129.4 \%$, $186.1 \%, 72.5 \%$ and $112.9 \%$, respectively, compared to the integrated counterparts. The snap-fitted BCC-Z lattice has the most increase in both compressive strength and energy absorption among these four configurations.

\subsection{Compressive responses of unit cells with different struts thickness}

Build orientation and imperfection on the surface (roughness) of the strut are the two key factors contribute to the mechanical performance of PolyJet struts. Previous research has shown that the roughness of struts is independent of the strut diameter and a change in diameter can largely affect the mechanical properties [28]. Fig. 8 shows the surface qualities of struts printed in $0^{\circ}$ (along the $\mathrm{X}$ direction), $45^{\circ}$ (along the $\mathrm{XZ}$ direction) and $90^{\circ}$ (along the $\mathrm{Z}$ direction) with three strut thicknesses, $t$ $=1.5 \mathrm{~mm}, t=2 \mathrm{~mm}$ and $t=2.5 \mathrm{~mm}$. Struts printed in $0^{\circ}$ have smooth surfaces for all the three scales (Fig. 8a). High surface roughness is observed at struts printed in $45^{\circ}$ and $90^{\circ}$. The roughness of $45^{\circ}$ is smaller (Fig. 8b), while the highest surface roughness is observed on struts printed in $90^{\circ}$ (Fig. 8c). Higher surface roughness leads to stress concentration and poor mechanical performance.

Four kinds of unit cells with square cross section struts of constant height, $H$, and various thicknesses, $t$, are made to enable study of the

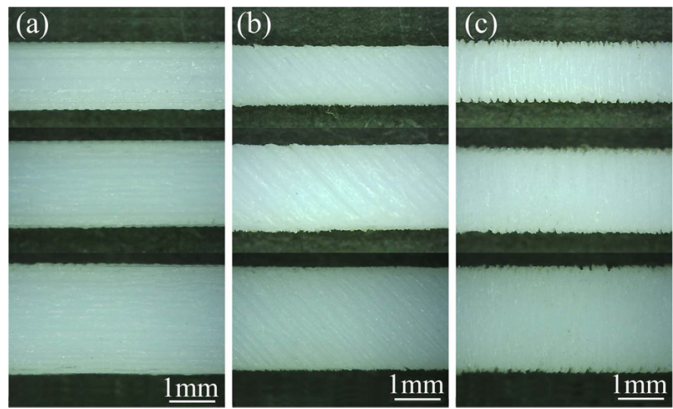

Fig. 8. Surface quality of struts printed in (a) $0^{\circ}$, (b) $45^{\circ}$ and (c) $90^{\circ}$ with strut thickness $t=1.5 \mathrm{~mm}, 2.0 \mathrm{~mm}$, and $2.5 \mathrm{~mm}$.

strut thickness upon compressive mechanical response. The unit cells with height, $H=34 \mathrm{~mm}$, and three strut thickness, $t=1.5 \mathrm{~mm}, 2 \mathrm{~mm}$ and $2.5 \mathrm{~mm}$, are fabricated by the snap-fit method and integrate method, respectively. Three samples of each strut thickness of the four kinds of lattices are tested to reduce scatter.

Comparisons of compressive failure modes and macroscopic stressstrain curves between snap-fitted and integrated lattices with three different strut thicknesses are shown in Figs. 9 and 10. Fig. 9(a) and Fig. 10(a) show the compressive failure modes and stress-strain curves of the integrated and snap-fitted BCC unit cells. The initial fracture of the integrated BCC cell with $t=1.5 \mathrm{~mm}$ appears in the center of a strut at a strain of 0.156 . For the integrated BCC cell with $t=2.0 \mathrm{~mm}$, one strut fails catastrophically at 0.071 strain. The failure mode is reflected in the stress-strain curve, which decreases in a stair-stepping way after the peak value. Catastrophic failure occurs in the integrated BCC lattice with $t=2.5 \mathrm{~mm}$ at a strain of 0.115 after the initial crack appearing at a strain of 0.103 , resulting in an immediate drop of stress to zero. By contrast, no catastrophic failure is observed in the snap-fitted BCC cells. There is no noticeable failure in the snap-fitted BCC cell with $t=1.5$ $\mathrm{mm}$ during the whole compressive process. Fractures of the snap-fitted BCC cell with $t=2.0 \mathrm{~mm}$ and $t=2.5 \mathrm{~mm}$ are initiated near the mediate node at strains of 0.288 and 0.147 , respectively. All the stressstrain curves of the snap-fitted BCC lattices decrease continuously after the peak stress. Fig. 9(b) and Fig. 10(b) show the compressive failure modes and stress-strain curves of the integrated and snap-fitted BCC-Z unit cells. Vertical struts in three scales of the integrated BCC-Z lattices have a similar failure mode. Negligible deformation of the struts is observed in the integrated BCC-Z cell until catastrophic strut failure was initiated in one vertical strut, and then the rest vertical struts fail in the same way in succession, resulting in a serrated decrease in the stressstrain curves. The failure mode of the remaining structure is similar to the integrated BCC cell. By contrast, global buckling and large 


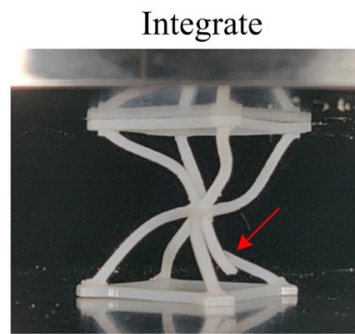

$t=1.5 \mathrm{~mm}, \varepsilon=0.156$

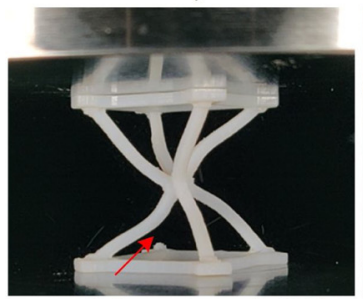

$t=2.0 \mathrm{~mm}, \varepsilon=0.071$

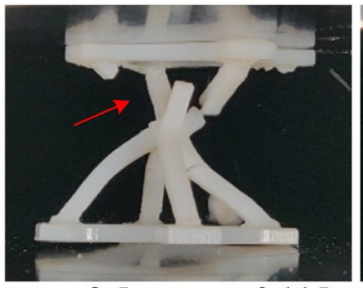

$t=2.5 \mathrm{~mm}, \varepsilon=0.115$
Snap-fit

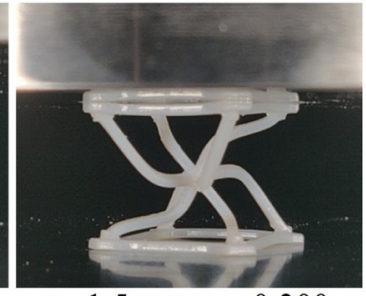

$t=1.5 \mathrm{~mm}, \varepsilon=0.300$

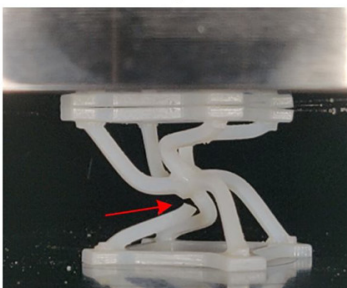

$t=2.0 \mathrm{~mm}, \varepsilon=0.288$

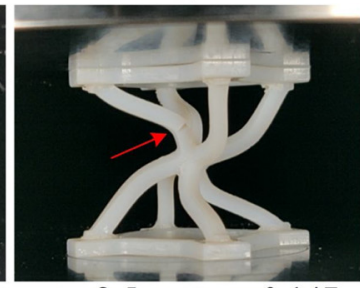

$t=2.5 \mathrm{~mm}, \varepsilon=0.147$

(a)

$10 \mathrm{~mm}$

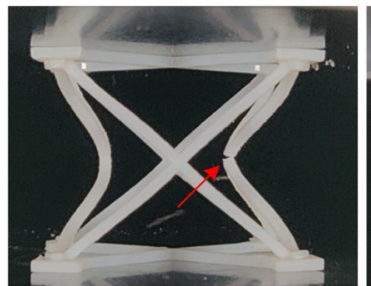

$t=1.5 \mathrm{~mm}, \varepsilon=0.076$

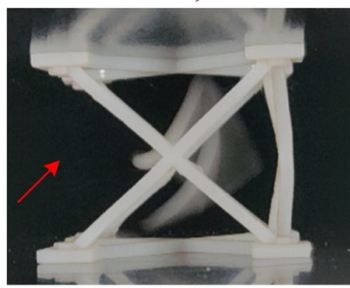

$t=2.0 \mathrm{~mm}, \varepsilon=0.041$

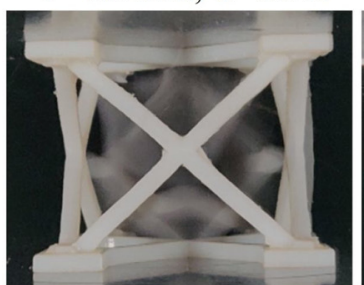

$t=2.5 \mathrm{~mm}, \varepsilon=0.038$
Snap-fit

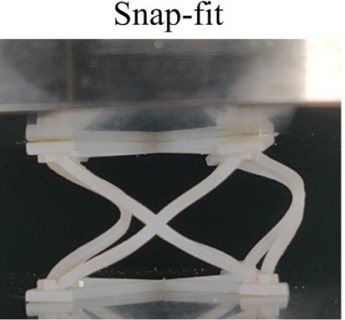

$t=1.5 \mathrm{~mm}, \varepsilon=0.300$

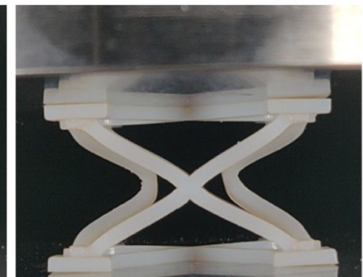

$t=2.0 \mathrm{~mm}, \varepsilon=0.300$

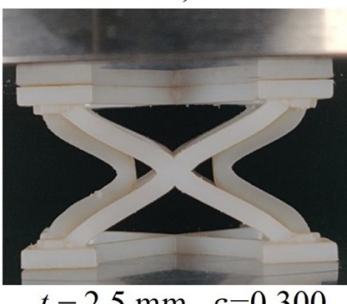

(c)

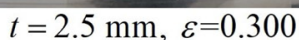

$10 \mathrm{~mm}$

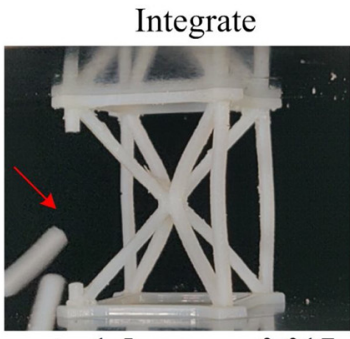

$t=1.5 \mathrm{~mm}, \varepsilon=0.017$

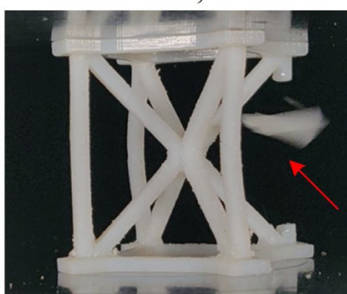

$t=2.0 \mathrm{~mm}, \varepsilon=0.017$

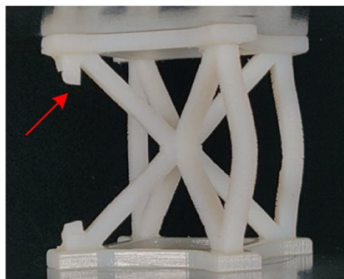

$t=2.5 \mathrm{~mm}, \varepsilon=0.032$
Snap-fit

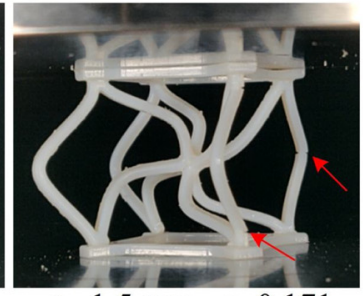

$t=1.5 \mathrm{~mm}, \quad \varepsilon=0.171$

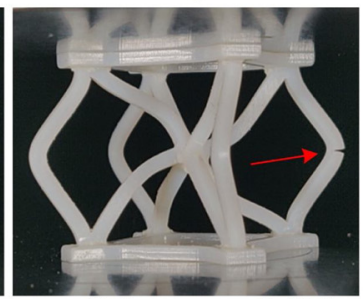

$t=2.0 \mathrm{~mm}, \varepsilon=0.138$

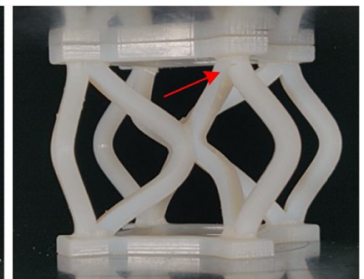

$t=2.5 \mathrm{~mm}, \varepsilon=0.126$

(b)

$10 \mathrm{~mm}$
Integrate

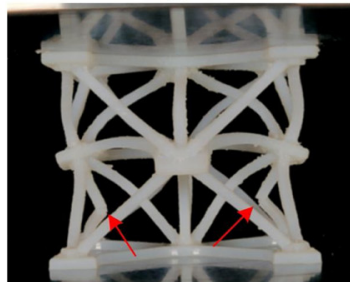

$t=1.5 \mathrm{~mm}, \varepsilon=0.082$

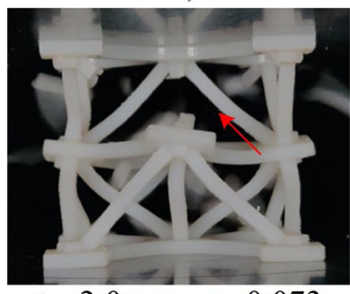

$t=2.0 \mathrm{~mm}, \varepsilon=0.073$

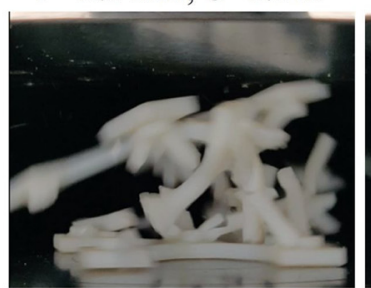

$t=2.5 \mathrm{~mm}, \varepsilon=0.106$

Snap-fit

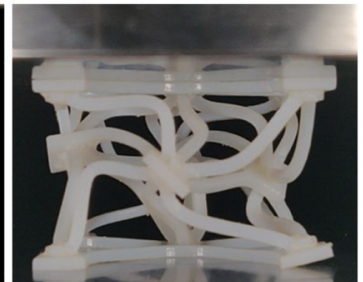

$t=1.5 \mathrm{~mm}, \varepsilon=0.300$

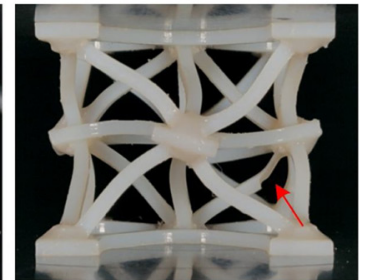

$t=2.0 \mathrm{~mm}, \varepsilon=0.076$

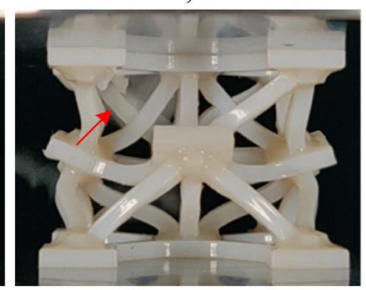

$t=2.5 \mathrm{~mm}, \varepsilon=0.132$

(d)

$10 \mathrm{~mm}$

Fig. 9. Failure modes of 3 scales integrated and snap-fitted unit cell (a)BCC lattice, (b) BCC-Z lattice, (c) FCC lattice and (d) octet lattice. 


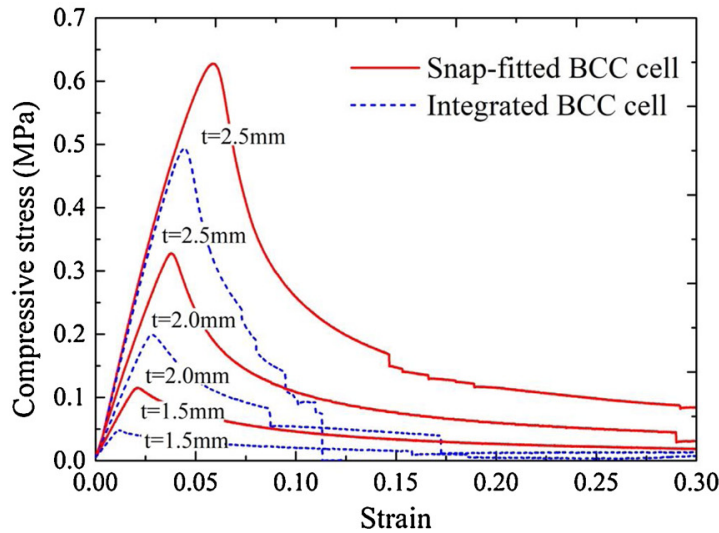

(a)

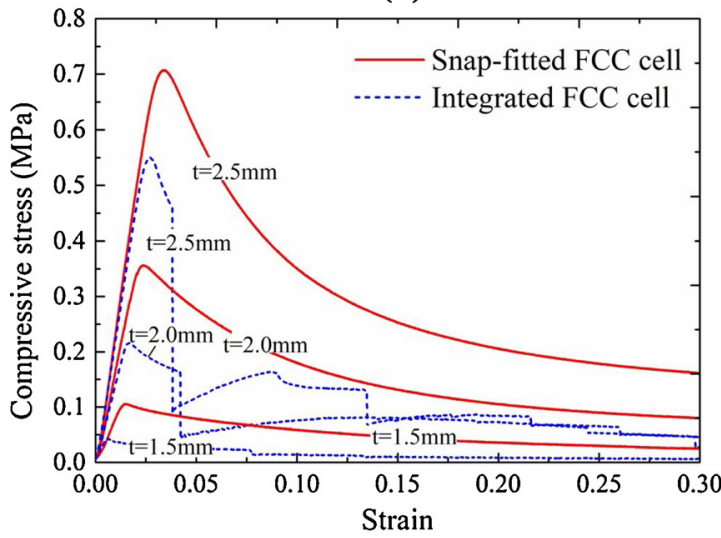

(c)

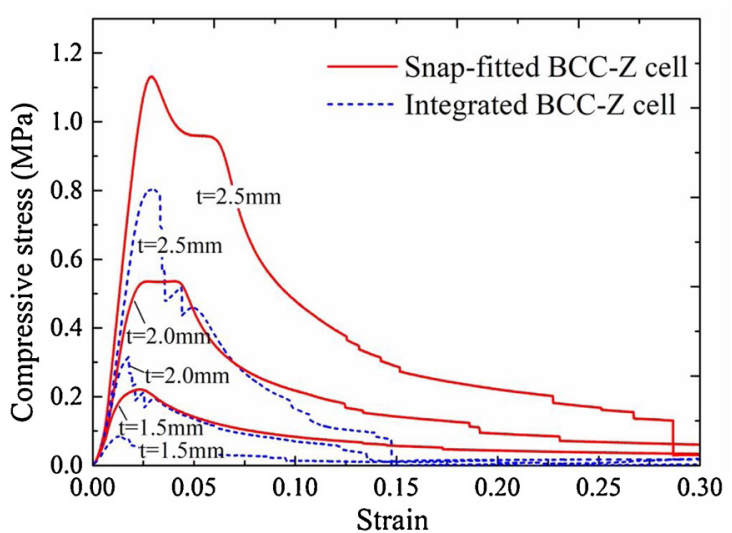

(b)

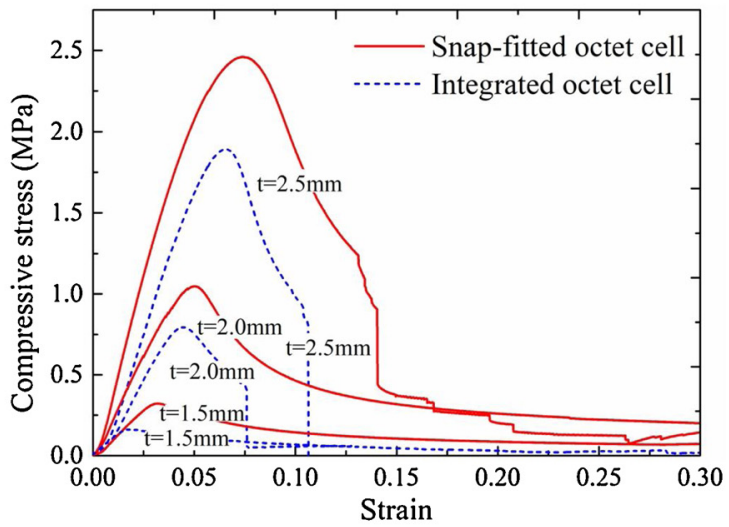

(d)

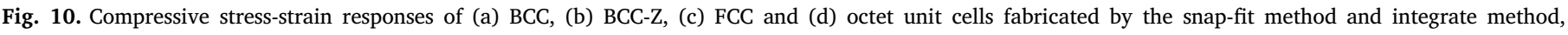
respectively, with strut thickness $t=1.5 \mathrm{~mm}, 2 \mathrm{~mm}$ and $2.5 \mathrm{~mm}$.

deformation of both the vertical and diagonal struts of the snap-fitted BCC-Z lattice is observed before failure. The initial failure of the snapfitted BCC-Z cells with strut scale $t=1.5 \mathrm{~mm}, 2.0 \mathrm{~mm}$ and $2.5 \mathrm{~mm}$ occurs at the middle and end of the vertical struts at strains of 0.171 , 0.138 and 0.126 , respectively. It is worth mentioning that the diagonal struts reach their critical buckling load after the vertical struts, delaying the stress drops as shown in Fig. 10(b). Fig. 9(c) and Fig. 10(c) show the compressive failure modes and stress-strain curves of the integrated and snap-fitted FCC unit cells. The integrated FCC unit cell with $t=1.5 \mathrm{~mm}$ shows an initial crack in the central cross part of struts at a strain of 0.076. With the increase of strut scale, one side of the integrated FCC cell fractures catastrophically at strains of 0.041 and 0.048 for $t=2.0$ $\mathrm{mm}$ and $t=2.5 \mathrm{~mm}$, respectively, resulting in a dramatic stress drop in the stress-strain curves. By contrast, the snap-fitted FCC unit cells deform uniformly without noticeable failure during the whole compressive process, resulting in a continuous decreasing curve after the peak value in the stress-strain curves. Fig. 9(d) and Fig. 10(d) show the compressive failure modes and stress-strain curves of the integrated and snap-fitted octet unit cells. The integrated octet unit cell tends to fail catastrophically as the strut thickness increases. Struts in the integrated octet cell with scale $t=1.5 \mathrm{~mm}$ break at the center of the struts at a strain of 0.082 . Several struts in the upper half of the integrated octet cell with scale $t=2 \mathrm{~mm}$ fail catastrophically at a strain of 0.073 , resulting in an immediate stress drop to a low value. The integrated octet cell with $t=2.5 \mathrm{~mm}$ catastrophic fails at a strain of 0.106 , resulting in an immediate stress drop to zero. By contrast, the snap-fitted octet cell with $t=1.5 \mathrm{~mm}$ deforms uniformly under the whole compressive process without noticeable failure. The initial fracture of the snap-fitted octet cell with $t=2 \mathrm{~mm}$ is observed at a strain of 0.076 at the end of one strut. The catastrophic failure of one strut of the snap-fitted cell with $t=2.5 \mathrm{~mm}$ occurs at a strain of 0.136 , inducing the stress drop into a low value.

With the increase of strut thickness, the failure mode of the integrated lattices tends to change from the gradual fracture of struts into catastrophic failure. Large struts deformation was observed in the snapfitted lattices before failure. The difference in failure modes between different struts thickness is not obvious in the snap-fitted lattices.

Comparisons of strengths between snap-fitted lattices and integrated lattices with three different strut scales are shown in Fig. 11. Strengths of the $1.5 \mathrm{~mm}, 2.0 \mathrm{~mm}$ and $2.5 \mathrm{~mm}$ snap-fitted unit lattices are increased by 131.9-164.9 \%, 45.9-81.8 \%, and 16.7-47\%, respectively, compared to the integrated counterparts of the four configurations. With the decrease of the strut thickness, all the configurations have a similar trend that the benefit on strength of the snap-fitted lattices becomes more pronounced. This is thought to be a consequence that the struts of the integrated lattices are more sensitive to imperfections as the decrease of struts scale, resulting in the dramatic reduction of lattices strength at small strut thickness. The best improvement in strength occurs in the snap-fitted BCC-Z configuration which is consistent with the experimental results of multi-cell lattices. This is thought to be a consequence that the vertical struts printed in $90^{\circ}$ in the integrated BCC-Z lattice have the poorest surface quality and mechanical properties. The vertical struts in the integrated BCC-Z lattice fail in succession at a small macroscopic strain while all the struts in the snap-fitted BCC-Z lattice undergo large deformation under compression. This difference can also explain why the snap-fitted BCC-Z lattice has the highest increase in energy absorption in the four kinds of configurations compared to the integrated counterpart (Fig. 7b). 


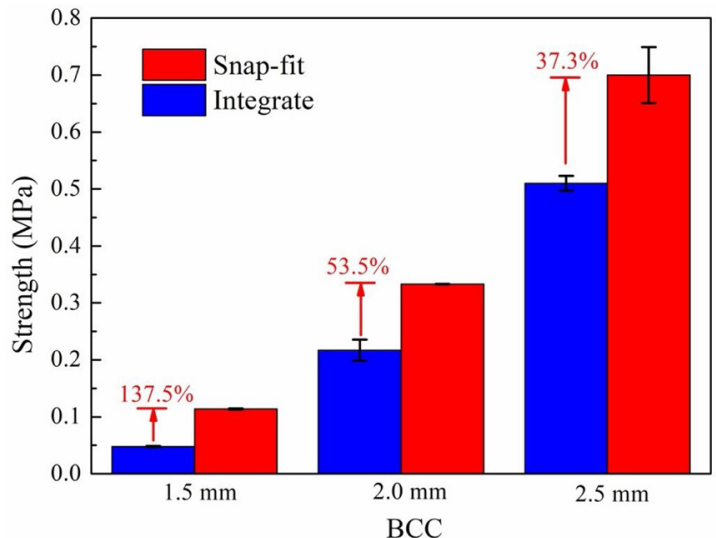

(a)

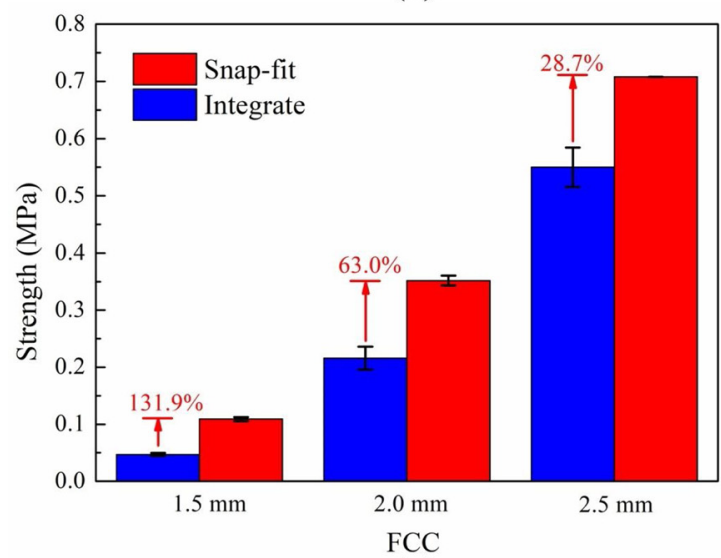

(c)

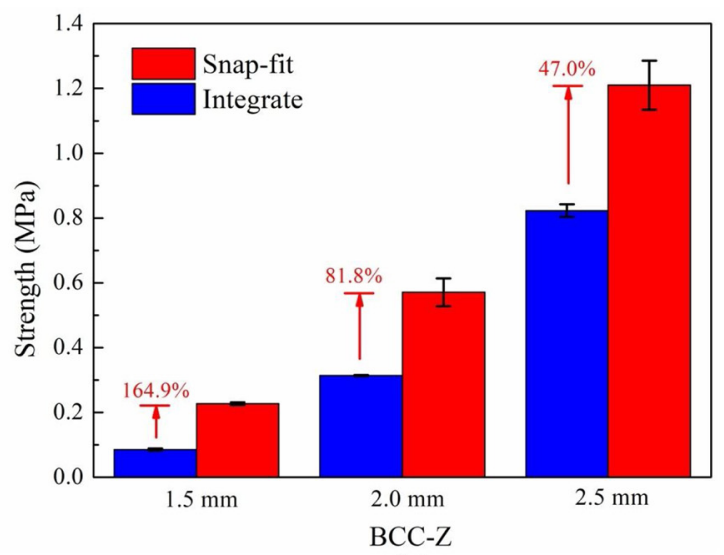

(b)

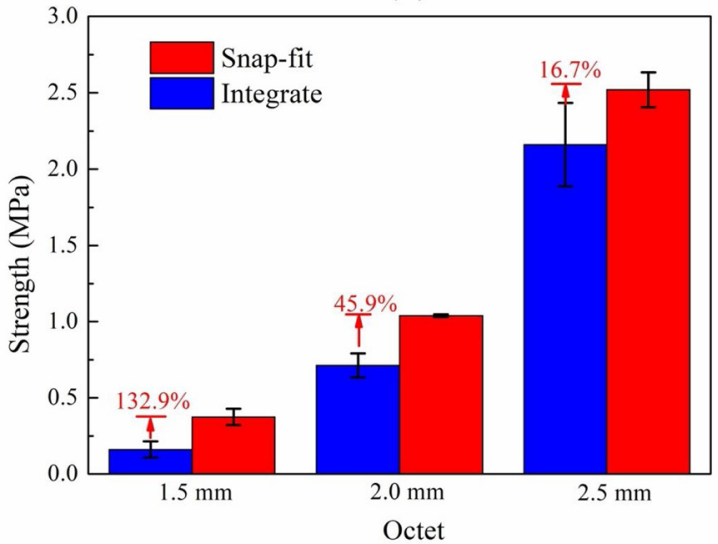

(d)

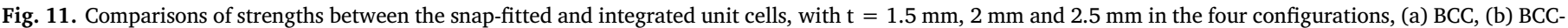
Z, (c) FCC and (d) octet.

\section{Analytical predictions and comparisons with experiments}

\subsection{Analytical models}

For the facility of fabrication and analysis, all the struts in these four kinds of unit cells have the same cross section. This also means the strength of the unit cell tested in this paper is higher than that of their multi-cell counterparts since the unit cell has no shared struts.

The effective peak strength of the octet lattice has been discussed in detail by Dong [29]. The analysis of BCC, BCC-Z and FCC cells is similar to the octet cell.

The peak strength, $\sigma_{p k}$, of the BCC unit cell is given by

$\sigma_{p k}=2 \sqrt{2} \frac{t^{2}}{A_{\text {cell }}^{B C C}} \sigma_{y}$

where $A_{\text {cell }}^{B C C}=(\sqrt{2} l+t+b)^{2}$ is the cross-sectional area of the BCC unit cell, referring to Fig. 2(a), and $\sigma_{y}$ is the compressive yielding strength of the model material.

The peak strength, $\sigma_{p k}$, of the BCC-Z unit cell is given by

$\sigma_{p k}=\left(4+\frac{2 H}{3 l}\right) \frac{t^{2}}{A_{\text {cell }}^{B C C-Z}} \sigma_{y}$

where $A_{\text {cell }}^{B C-Z}=(\sqrt{2} l+t+b)^{2}$ is the cross-sectional area of the BCC-Z unit cell and $H=\sqrt{2} l+2 t$ is the height of the unit cell, referring to Fig. 2(b).

The peak strength, $\sigma_{p k}$, of the FCC unit cell is given by

$\sigma_{p k}=4 \sqrt{2} \frac{t^{2}}{A_{\text {cell }}^{\text {FCC }}} \sigma_{y}$ where $A_{\text {cell }}^{F C C}=(\sqrt{2} l+b)^{2}$ is the cross-sectional area of the FCC unit cell, referring to Fig. 2(c).

The peak strength, $\sigma_{p k}$, of the octet unit cell is given by

$\sigma_{p k}=6 \sqrt{2} \frac{t^{2}}{A_{\text {cell }}^{\text {octet }}} \sigma_{y}$

where $A_{\text {cell }}^{\text {octet }}=(\sqrt{2} l+t+b)^{2}$ is the cross-sectional area of the octet unit cell, referring Fig. 2(d).

When the struts collapse by elastic buckling prior to plastic yielding, the lattice strength is obtained by replacing the yield strength, $\sigma_{y}$, with the elastic buckling stress. The elastic buckling stress of struts in BCC and octet lattices is given by

$\sigma_{E}=\frac{k^{2} \pi^{2} E_{S}}{12}\left(\frac{t}{l}\right)^{2}$

The elastic buckling stress of struts in BCC-Z lattice is given by

$\sigma_{E}=\frac{k^{2} \pi^{2} E_{S}}{12}\left(\frac{t}{H}\right)^{2}$

The cross joint in FCC truss has negligible constraint on the out-of-plane buckling deformantion of strut. Thus, the effective length of buckling deformation of strut in FCC lattice is $2 l$. The elastic buckling stress of struts in FCC lattice is given by

$\sigma_{E}=\frac{k^{2} \pi^{2} E_{S}}{12}\left(\frac{t}{2 l}\right)^{2}$

Where $E_{s}$ is elastic modulus of the model material and the $k$ is determined by the end conditions on the buckling struts: $k^{2}=1$ and 4 for pin-jointed and built-in end conditions, respectively. If one end of the strut is pin-jointed and the other is a built-condition, $k^{2} \approx 2$. 


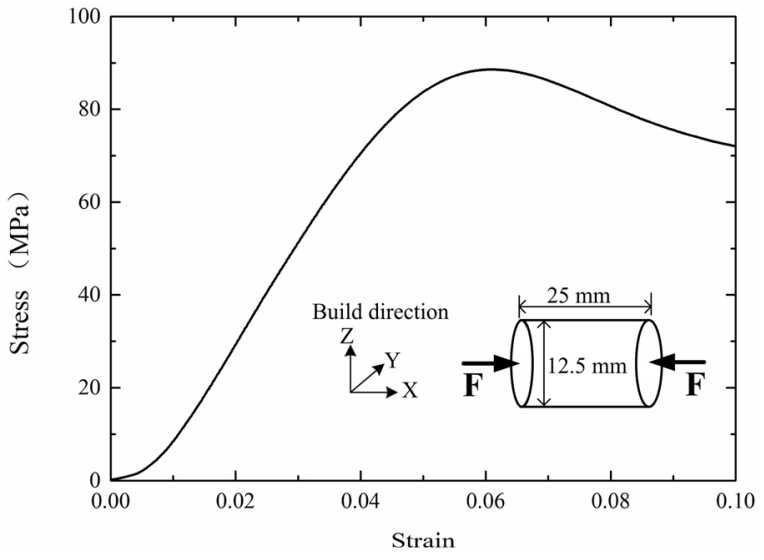

Fig. 12. The compressive stress versus strain responses for VeroWhitePlus (RGD835) sample printed in $0^{\circ}$ (along the $\mathrm{X}$ direction). The elastic modulus and yield strength are $2240 \mathrm{MPa}$ and $88.5 \mathrm{MPa}$, respectively.

When struts in the lattice buckle between the proportional limit and yield stress of the solid material, the compressive strength of the lattice is controlled by inelastic buckling. The inelastic buckling stress, $\sigma_{I E}$, is found by replacing elastic modulus, $E_{s}$ in Eq. (6), (7) and (8), with tangent modulus $E_{t}$ [30].

The inelastic buckling stress of struts in BCC and octet lattices is given by

$\sigma_{I E}=\frac{k^{2} \pi^{2} E_{t}}{12}\left(\frac{t}{l}\right)^{2}$

The inelastic buckling stress of struts in BCC-Z lattice is given by

$\sigma_{I E}=\frac{k^{2} \pi^{2} E_{t}}{12}\left(\frac{t}{H}\right)^{2}$

The inelastic buckling stress of struts in FCC lattices is given by

$\sigma_{I E}=\frac{k^{2} \pi^{2} E_{t}}{12}\left(\frac{t}{2 l}\right)^{2}$

\subsection{Modeling material properties}

In order to compare the measured strengths of both the snap-fitted and integrated unit cells with the maximum predicted values of strength, the solid cylindrical sample printed in $0^{\circ}$ (along $\mathrm{X}$ direction) is tested in compression at speed $1.3 \mathrm{~mm} / \mathrm{min}$. The stress-strain curve of modeling material, VeroWhitePlus (RGD835), is plotted in Fig. 12. The measured elastic modulus and yield strength are $2240 \mathrm{MPa}$ and 88.5 $\mathrm{MPa}$, respectively.

\subsection{Comparisons with predictions}

Comparisons of compressive strengths between predictions and measurements in the snap-fitted and integrated unit cells with the BCC, BCC-Z, FCC and octet configurations are shown in Fig. 13(a), (b), (c) and (d), respectively. The visible rotation of the intermediate node in BCC, FCC and octet lattices appears during the compressive process and thus we assume $k^{2} \approx 2$ in Eq. (6) and Eq. (9). We assume the vertical struts in BCC-Z lattice and struts in FCC lattice are built-in condition and thus take $k^{2}=4$ in Eq. (7), (8), (10) and (11). The yield strength, $\sigma_{y}$, and elastic modulus, $E_{\mathrm{s}}$, of the model material are taken as $88.5 \mathrm{MPa}$ and $2240 \mathrm{MPa}$, respectively. The compressive strengths of all three strut thicknesses of the snap-fitted unit cells for the four kinds of configurations are captured by the elastic buckling and inelastic buckling models. While the compressive strengths of all the integrated lattices are far less than the predictions. It worth mentioning that the models slightly over-predict the compressive peak strengths of the snap-fitted
BCC, FCC and octet unit cells. There are several reasons contributing to this slight over-prediction. The previous study has revealed that the mechanical properties of the PolyJet sample decrease with the diameter of the printed sample [28]. While the thickness of struts of the tested lattices is far less than the diameter of the solid cylindrical sample tested in this paper. Another reason is that although the strength and modulus in are assumed isotropic, actually, the strength and elastic modulus of the sample printed in the $\mathrm{X}$ direction are slightly larger than the other direction in the $\mathrm{X}-\mathrm{Y}$ plane $[19,28]$. The build direction of half struts in the snap-fitted BCC, FCC and octet cells is along the X direction and half is along the $\mathrm{Y}$ direction. The yield strength and elastic modulus in prediction are taken to be the value of the X-direction sample. Imperfections at the bonded nodes from assembly may result in stress concentration and strength decrease.

\section{Discussion}

The present work has revealed that the polymer lattices fabricated by the PolyJet technology and snap-fit method have almost optimal mechanical properties and no problem of removing support material. There are also several things worth noting in the snap-fit method.

The snap-fit assembly method may bring in assembly errors at the bonded nodes, which could result in strain incompatibility and stress concentration at the nodal regions under compression, especially for the lattices which have a large twist deformation at the bonded nodes such as the octet lattice as shown in Figs. 6(d) and 9 (d). Thus, the bond of the connected nodes in the snap-fitted lattices is important to the mechanical performance of the lattices. Epoxy glue with competent bonded strength and good toughness is used in this work to bond the nodes of the snap-fitted lattices, which can effectively reduce the strain incompatibility and stress concentration at the connected nodes, providing a large failure strain and high strength. The anisotropy of mechanical properties only depends on the printing technology and has nothing to do with the printing materials, which means the snap-fit method can be used in other printing materials of the PolyJet technology. However, the higher strength and stiffness of the model material, the higher strain incompatibility and stress concentration may occur at the adhesive nodes. Thus, a higher strength and toughness adhesive need to be chosen to alleviate this problem.

Since all the struts in the snap-fitted lattices are printed in the $\mathrm{X}-\mathrm{Y}$ plane and almost have the same mechanical properties, the snap-fitted lattices could achieve the macroscopic isotropic when the lattices satisfy the geometric symmetry. However, the lattices fabricated in this paper do not strictly satisfy the geometric symmetry and have only been tested in one direction. The snap-fitted lattices with geometric symmetry and their compressive tests in the other directions will be designed and studied in the future work.

\section{Conclusions}

The snap-fit method is introduced into PolyJet technology to fabricate polymer lattice structures to obtain maximum mechanical performance and minimum consumption of support material. Four kinds of $2 \times 2 \times 2$ cells lattices with strut thickness $t=2 \mathrm{~mm}$, namely BCC, BCC-Z, FCC, and octet, are design and fabricated with the snap-fit method and conventional integrate method. Both of printing material consumption and printing time are reduced by over $80 \%$ in the novel method, compared to the conventional integrated printing method. The strengths of the snap-fitted BCC, BCC-Z, FCC and octet lattices increased by $102.3 \%, 121.9 \%, 107.0 \%$ and $105.5 \%$, respectively, and the specific energy absorptions increased by $129.4 \%, 186.1 \%, 72.5 \%$ and $112.9 \%$, respectively, compared to the integrated counterparts.

Unit lattice cells of the four kinds of configurations with three different strut thicknesses are fabricated to investigate the effect of strut thickness on mechanical performance. Strengths of the $1.5 \mathrm{~mm}, 2.0 \mathrm{~mm}$ and $2.5 \mathrm{~mm}$ snap-fitted unit lattices are increased by $131.9-164.9 \%$, 


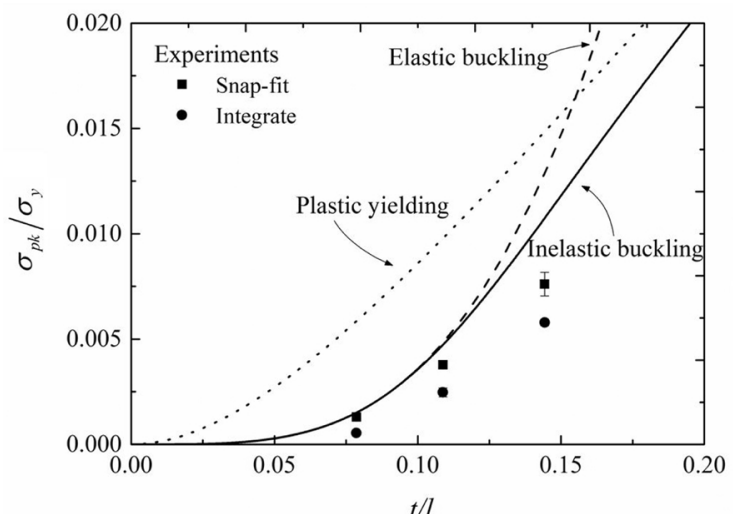

(a)

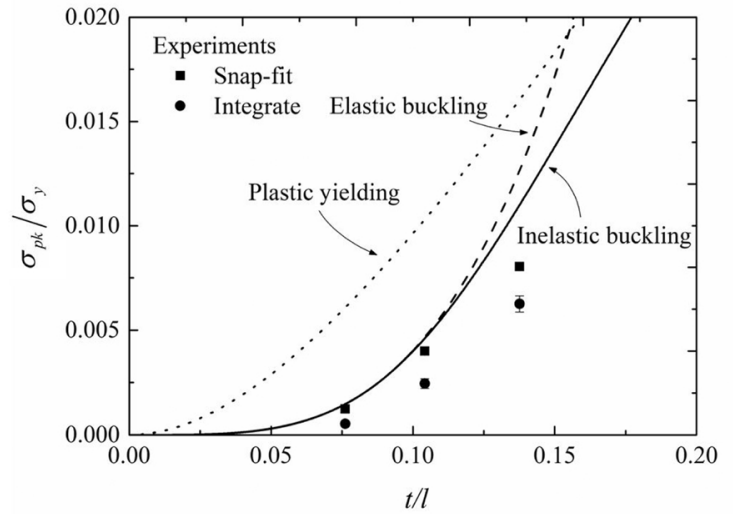

(c)

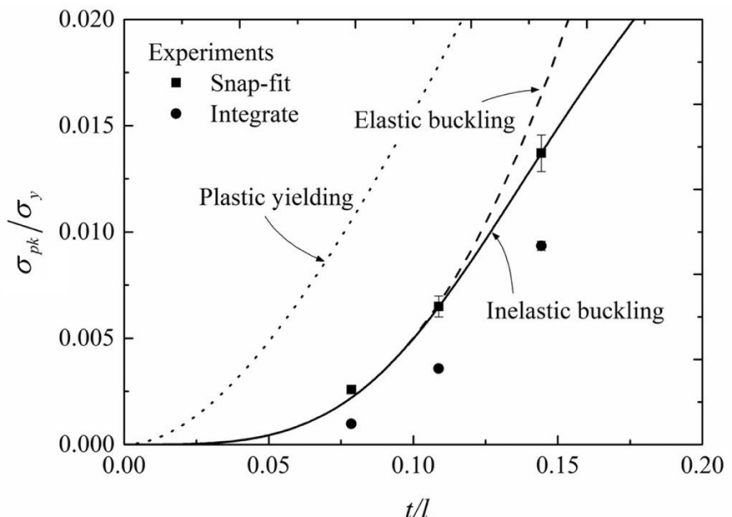

(b)

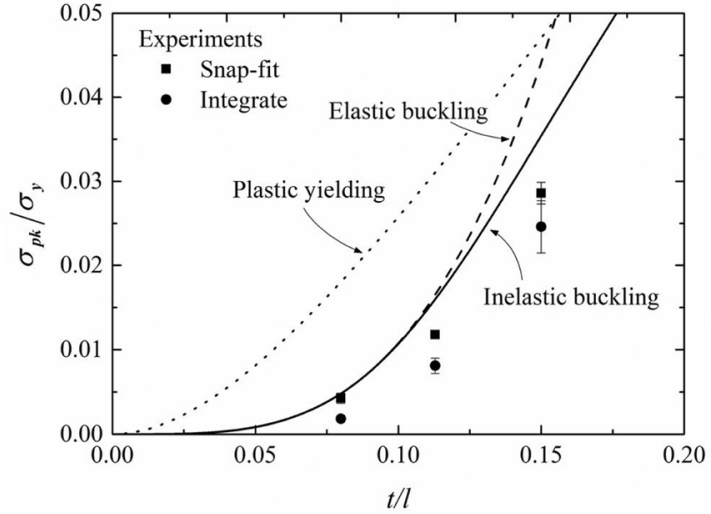

(d)

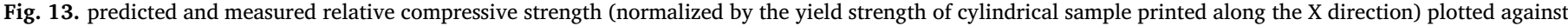
strut slenderness ratio $t / l$ of the (a) BCC, (b) BCC-Z, (c) FCC and (d) octet unit cells.

45.9-81.8 \%, and 16.7-47\%, respectively, compared to the integrated counterparts of the four configurations. With the decrease of strut thickness, the benefit on strength of the snap-fitted lattices becomes more pronounced. Compared with analytical predictions, the measured peak strengths of snap-fitted lattices are very close to the theoretical maximum, whereas the measured strengths of integrated lattices are far low from those theoretical values.

This study opens up an avenue for the fabrication of large scale 3D printed lattice structures with optimal mechanical properties and without support material removal problem.

\section{Declaration of interests}

The authors declare that they have no known competing financial interests or personal relationships that could have appeared to influence the work reported in this paper.

\section{CRediT authorship contribution statement}

Wenfeng Liu: Conceptualization, Methodology, Investigation, Writing - original draft, Writing - review \& editing. Hongwei Song: Methodology, Writing - review \& editing, Supervision, Project administration, Funding acquisition. Chenguang Huang: Writing - review \& editing, Supervision, Project administration, Funding acquisition.

\section{Acknowledgment}

Financial supports from National Natural Science Foundation of China (Grant Nos. 91016025, 11472276, 11602271 and 11332011) are gratefully acknowledged.

\section{Appendix A. Supplementary data}

Supplementary material related to this article can be found, in the online version, at doi:https://doi.org/10.1016/j.addma.2020.101257.

\section{References}

[1] T.A. Schaedler, A.J. Jacobsen, A. Torrents, A.E. Sorensen, J. Lian, J.R. Greer, L. Valdevit, W.B. Carter, Ultralight metallic microlattices, Science 334 (6058) (2011) 962-965.

[2] S.J. Yeo, M.J. Oh, P.J. Yoo, Structurally controlled cellular architectures for highperformance ultra-lightweight materials, Adv. Mater. (2018) e1803670.

[3] L.R. Meza, S. Das, J.R. Greer, Strong, lightweight, and recoverable three-dimensional ceramic nanolattices, Science 345 (6202) (2014) 1322-1326.

[4] T. Frenzel, C. Findeisen, M. Kadic, P. Gumbsch, M. Wegener, Tailored buckling microlattices as reusable light-weight shock absorbers, Adv. Mater. 28 (28) (2016) 5865-5870.

[5] S. Shan, S.H. Kang, J.R. Raney, P. Wang, L. Fang, F. Candido, J.A. Lewis, K. Bertoldi, Multistable architected materials for trapping elastic strain energy, Adv. Mater. 27 (29) (2015) 4296-4301.

[6] H.M.A. Kolken, S. Janbaz, S.M.A. Leeflang, K. Lietaert, H.H. Weinans, A.A. Zadpoor, Rationally designed meta-implants: a combination of auxetic and conventional meta-biomaterials, Mater. Horiz. 5 (1) (2018) 28-35.

[7] A.A. Zadpoor, Meta-biomaterials, Biomater. Sci. 8 (1) (2019) 18-38.

[8] T. Tancogne-Dejean, A.B. Spierings, D. Mohr, Additively-manufactured metallic micro-lattice materials for high specific energy absorption under static and dynamic loading, Acta Mater. 116 (2016) 14-28.

[9] M. Santorinaios, W. Brooks, C.J. Sutcliffe, R.A.W. Mines, Crush Behaviour of Open Cellular Lattice Structures Manufactured Using Selective Laser Melting 1 (2006), pp. 481-490.

[10] Z. Ozdemir, E. Hernandez-Nava, A. Tyas, J.A. Warren, S.D. Fay, R. Goodall, I. Todd, H. Askes, Energy absorption in lattice structures in dynamics: experiments, Int. J. Impact Eng. 89 (2016) 49-61.

[11] S. McKown, Y. Shen, W.K. Brookes, C.J. Sutcliffe, W.J. Cantwell, G.S. Langdon, G.N. Nurick, M.D. Theobald, The quasi-static and blast loading response of lattice structures, Int. J. Impact Eng. 35 (8) (2008) 795-810.

[12] Y. Xu, H. Zhang, B. Šavija, S. Chaves Figueiredo, E. Schlangen, Deformation and fracture of 3D printed disordered lattice materials: experiments and modeling, 
Mater. Des. 162 (2019) 143-153.

[13] M.R. Karamooz Ravari, M. Kadkhodaei, M. Badrossamay, R. Rezaei, Numerical investigation on mechanical properties of cellular lattice structures fabricated by fused deposition modeling, Int. J. Mech. Sci. 88 (2014) 154-161.

[14] M. Kaur, T.G. Yun, S.M. Han, E.L. Thomas, W.S. Kim, 3D printed stretching-dominated micro-trusses, Mater. Des. 134 (2017) 272-280.

[15] G. Dong, G. Wijaya, Y. Tang, Y.F. Zhao, Optimizing process parameters of fused deposition modeling by Taguchi method for the fabrication of lattice structures, Addit. Manuf. 19 (2018) 62-72.

[16] P. Egan, X. Wang, H. Greutert, K. Shea, K. Wuertz-Kozak, S. Ferguson, Mechanical and biological characterization of 3D printed lattices, 3D Print. Addit. Manuf. 6 (2) (2019) 73-81.

[17] P.F. Egan, I. Bauer, K. Shea, S.J. Ferguson, Mechanics of three-dimensional printed lattices for biomedical devices, J. Mech. Des. 141 (3) (2019).

[18] J. Mueller, K. Shea, Stepwise graded struts for maximizing energy absorption in lattices, Extreme Mech. Lett. 25 (2018) 7-15.

[19] J. Mueller, K. Shea, C. Daraio, Mechanical properties of parts fabricated with inkjet 3D printing through efficient experimental design, Mater. Des. 86 (2015) 902-912.

[20] S.H. Ahn, M. Montero, D. Odell, S. Roundy, P.K. Wright, Anisotropic material properties of fused deposition modeling ABS, Rapid Prototyp. J. 8 (4) (2002) 248-257.

[21] M. Suard, G. Martin, P. Lhuissier, R. Dendievel, F. Vignat, J.J. Blandin, F. Villeneuve, Mechanical equivalent diameter of single struts for the stiffness prediction of lattice structures produced by electron beam melting, Addit. Manuf. 8
(2015) 124-131.

[22] R. Gautam, S. Idapalapati, S. Feih, Printing and characterisation of Kagome lattice structures by fused deposition modelling, Mater. Des. 137 (2018) 266-275.

[23] R. Wauthle, B. Vrancken, B. Beynaerts, K. Jorissen, J. Schrooten, J.-P. Kruth, J. Van Humbeeck, Effects of build orientation and heat treatment on the microstructure and mechanical properties of selective laser melted Ti6Al4V lattice structures, Addit. Manuf. 5 (2015) 77-84.

[24] T. Stanković, J. Mueller, K. Shea, The effect of anisotropy on the optimization of additively manufactured lattice structures, Addit. Manuf. 17 (2017) 67-76.

[25] W. Liu, H. Song, Z. Wang, J. Wang, C. Huang, Improving mechanical performance of fused deposition modeling lattice structures by a snap-fitting method, Mater. Des. 181 (2019) 108065.

[26] Stratasys Ltd., What is PolyJet Technology? https://www.stratasys.com/ polyjettechnology. (Accessed 24 January).

[27] J. Mueller, K. Shea, The effect of build orientation on the mechanical properties in inkjet 3D-printing, International Solid Freeform Fabrication Symposium (2015) 983-992.

[28] J. Mueller, K. Shea, Buckling, build orientation, and scaling effects in 3D printed lattices, Mater. Today Commun. 17 (2018) 69-75.

[29] L. Dong, V. Deshpande, H. Wadley, Mechanical response of Ti-6Al-4V octet-truss lattice structures, Int. J. Solids Struct. 60-61 (2015) 107-124.

[30] S.P. Timoshenko, J.M. Gere, W. Prager, Theory of elastic stability, second edition, J. Appl. Mech. 29 (1) (1962) 220. 